\title{
Effect of cypermethrin insecticide on the microbial community in cucumber phyllosphere
}

\author{
ZHANG Baoguo ${ }^{1,3}$, ZHANG Hongxun ${ }^{1,3}$, JIN Bo $^{2}$, TANG Ling ${ }^{1}$, YANG Jianzhou ${ }^{1}$, LI Baoju ${ }^{4}$, \\ ZHUANG Guoqiang ${ }^{1}$, BAI Zhihui ${ }^{1,2, *}$ \\ 1. Research Centre for Eco-Environmental Sciences, Chinese Academy of Sciences, Beijing 100085, China. E-mail: baoguozhang521 @yahoo.com.cn \\ 2. School of Earth and Environmental Science, University of Adelaide, Adelaide, SA 5005, Australia \\ 3. Graduate University of Chinese Academy of Sciences, Beijing 100085, China \\ 4. Institute of Vegetables and Flowers, Chinese Academy of Agricultural Sciences, Beijing 100081, China
}

Received 25 December 2007; revised 30 January 2008; accepted 12 March 2008

\begin{abstract}
Cucumber (Cucumis sativus) is one of the most widely used vegetable in the world, and different pesticides have been extensively used for controlling the insects and disease pathogens of this plant. However, little is known about how the pesticides affect the microbial community in cucumber phyllosphere. This study was the first attempt to assess the impact of pyrethroid insecticide cyperemethrin on the microbial communities of cucumber phyllosphere using biochemical and genetic approaches. Phospholipid fatty acid (PLFA) assay indicated that cyperemethrin insecticide treatment led to a significant increase in both total and bacterial biomass and a decrease in fungal biomass and the ratio of Gram-positive (GP) bacteria to Gram-negative (GN) bacteria within the cucumber phyllosphere. Principal-component analyses (PCA) suggested that the number of unsaturated and cyclopropane PLFAs (16:1 $\omega 9 \mathrm{t}$, 18:1 $\omega 7$, cy17:0, cy19:0) increased with the insecticide treatment, whereas the saturated PLFA i16:0, i17:0 decreased. The increase of GN bacteria implied that the cypermethrin insecticide might be a nutrient for the growth of these phyllosphere microbes. Terminal restriction fragment length polymorphism (T-RFLP) reinforced the PLFA results. A significant change of bacterial community structure was observed in the separate dendrogram cluster between control and treated samples with the cucumber phyllosphere following cypermethrin insecticide treatment. Moreover, the increased terminal restriction fragments (T-RFs) (58, 62, 89, 99, 119, 195, 239, 311,340 , and $473 \mathrm{bp}$ ) indicated that some bacteria might play a significant role in the insecticide degradation within the cucumber phylosphere, whereas the disappeared T-RFs $(44,51,96,223,306$, and $338 \mathrm{bp})$ implied that some other bacteria might potentially serve as microbial indicator of cyperemethrin insecticide exposure.
\end{abstract}

Key words: cucumber; cypermethrin insecticide; microbial community; phyllosphere; phospholipid fatty acid (PLFA); T-RFLP

\section{Introduction}

Aerial plant surfaces are generally colonized by large numbers of different microorganisms including bacteria, filamentous fungi, yeasts, and algae. The aerial habitat colonized by these microbes is termed as phyllosphere, and the inhabitants are called phyllosphere microbes (Lindow and Brandl, 2003). Microorganisms in the phyllosphere may include those that are pathogenic to the plant and nonpathogenic organisms, which play an important role in altering plant surface properties, fixating nitrogen, promoting the growth of plants, controlling the plant pathogens, degrading organic pollutants, and being an ideal monitors of plants pollution (Kvasnikov et al., 1974; Murty, 1984; Hirano and Upper, 2000; Krechel et al., 2002; Krimm et al., 2005; Schreiber et al., 2005; Sandhu et al., 2007). Although these phyllosphere microbes can grow through the utilization of the limited resources available in this

\footnotetext{
* Corresponding author. E-mail: zhbai@rcees.ac.cn.
}

habit, some environmental stress conditions, including fluctuation of water availability, temperature, nutrient supply, osmotic pressure, dose of UV radiation, and pollutant, can affect the phyllosphere microbial populations (Lindow and Leveau, 2002).

Insecticides have become an important tool to protect crops from insect and disease. However, these insecticides could also affect nontarget microorganisms on the plants phyllosphere. Impact of an insecticide on microorganisms depends upon the properties and the concentration of the insecticide, the type of the microorganisms, and the environment in which the microorganisms are growing (Lal and Saxena, 1982). Therefore, the population of microbial communities may vary in association with the addition of insecticide. Many studies have been carried out to assess the effects of insecticide on microorganism communities in different environment. Ko and Lockwood (1968) demonstrated an inhibitory effect of $10 \mathrm{mg} / \mathrm{L}$ DDT on several microorganisms. In contrast, Salonlus (1972) found that massive doses of DDT $\left(112 \mathrm{~kg} / \mathrm{hm}^{2}\right)$ did not alter the 
numbers of soil microflora. Chinalia and Killham (2006) reported that the microbial communities varied significantly as a result of addition of different amounts of 2,4-D in river sediments. Pandey and Chauhan (2007) found that both pesticides (Endo sulfan and Quinal phos) negatively affected the microbial biomass and the microbial activity. Vig et al. (2007) showed that successive applications of the six insecticides caused only short-lived adverse effects on the soil microorganisms in cotton fields and none of the insecticides used had any adverse effects on the soil fungi as reflected by their total numbers. Recently, Walter et al. (2007) reported that agrichemicals clearly affected nontarget, culturable apple phyllosphere microorganisms. Despite the frequent occurrence of insecticides in the phyllosphere environment of plants, little is known about their interactions with nontarget phyllosphere microorganisms.

Traditional culture-dependent techniques have limited application for the analysis of microbial assemblages since more than $90 \%$ of indigenous microorganisms disappeared in natural cultivation (Amann et al., 1995). In recent years, the culture-independent methods including Phospholipid fatty acid analysis (PLFA) and Terminal restriction fragment length polymorphism (T-RFLP) have been developed and used as important tools for the study of microbial diversity and community structure in the environments such as soils, water, activated sludge, and the rhizosphere of plants (Hiraishi et al., 2000; Xue et al., 2005; Ratcliff et al., 2006; Pang et al., 2007). However, a little information was reported in the literature to use these two cultureindependent methods to study the community structure of the phyllosphere. Moreover, applying these cultureindependent methods in ecotoxicology may be a new promising approach to detect pesticide-induced changes in microbial communities.

Cucumber (Cucumis sativus) is one of the most widely used vegetables in the world. During its growing process, the cucumber may get damaged by many insects and pathogens such as Pieris rapae L., Plutella xylostella L., Chrysodeoxis chalcites, Spodoptera litura F., Lipaphis erysim, Aphis gossypii, and Myzus persicae (Lenteren, 2000; Zhang et al., 2007). A number of insecticides are applied to control these insect pests of the cucumber. The objective of this study was to investigate and compare the effects of cypermethrin application on cucumber phyllosphere microbial community using PLFA and T-RFLP techniques.

\section{Materials and methods}

\subsection{Experimental design}

The experiments were conducted in a greenhouse located on the farm of Institute of Vegetables and Flowers, Chinese Academy of Agriculture Sciences, Beijing, China. The cucumber was grown in $110 \mathrm{~m}^{2}$ plots in a greenhouse on 10 April 2007. During the course of the experiment, the daily air temperature varied in a range between 18 and $26^{\circ} \mathrm{C}$, and the average relative air humidity was $63 \%$. The cucumber grew under the same local cultivation prac- tices, such as irrigation and fertilization. When the sixth leaf emerged, the plants were sprayed with 1:500 waterdiluted emulsifiable concentrated cypermethrin $(2.5 \%$, $W / W$ ), which is a recommended dosage for the cucumber. No pesticide was used during the whole growth period of the cucumber in a control experiment. Fifty gram of leaf material was collected from each control and treatment samples on day $0,1,3,6,10,15$ after treatment. Three replication of each sample were included. After picking, the samples were immediately put into sterile stomacher bags and transported to the laboratory at $4{ }^{\circ} \mathrm{C}$. The PLFA analysis was conducted directly. The leaves used for DNA extraction and T-RFLP were stored at $-20^{\circ} \mathrm{C}$ until analysis.

\subsection{Microorganism cell extraction}

The leave samples were collected aseptically using flame-sterilized forceps and placed in polypropylene tubes containing washing buffer $(0.1 \mathrm{~mol} / \mathrm{L}$ potassium phosphate buffer, $\mathrm{pH} \mathrm{7.0)}$ and sonicated for $7 \mathrm{~min}$ in an ultrasonic cleaning bath to dislodge microorganisms from leaves. Leave debris was removed by a low speed centrifugation ( $5 \mathrm{~min}, 500 \mathrm{~g}, 4^{\circ} \mathrm{C}$ ). The microbial cell suspension was centrifuged at $7,000 \times g$ for $15 \mathrm{~min}$ at $4^{\circ} \mathrm{C}$. Microorganism cells were resuspended in washing solution and frozen at $-20^{\circ} \mathrm{C}$ until processing.

\subsection{Phospholipid fatty acid analysis (PLFAs)}

Triplicate sample from the control and cypermethrintreated cucumber plants incubated for $0,1,3,6,10,15 \mathrm{~d}$ were randomly selected for PLFA analysis. Phospholipid fatty acids were extracted using a modified procedure described by Pennanen et al. (1999). In brief, $0.5 \mathrm{~g}$ of material washed from the surface of the leaves was transferred to a glass tube with a Teflon-lined screw cap. Lipids were extracted by a one-phase chloroform, methanol, and $0.15 \mathrm{~mol} / \mathrm{L}$ citrate buffer extractant. The total lipid extract was fractioned into neutral, glyco- and polar (phospho-) lipids by silicic acid chromatography. The methyl ester of nonadecanoic acid (19:0) was added as internal standard immediately before the separated phospholipids fatty acid was transesterified into fatty acid methyl esters using a mild alkaline methanolysis reaction. Fatty acid methyl esters (FAMEs) were analyzed using a GC-MS system (Hewlett Packard HP 6890, USA) equipped with a HP5 capillary column $(60 \mathrm{~m} \times 0.32 \mathrm{~mm})$. Total quantity (nmol/g) was determined using methyl ester of nonadecanoic acid as an internal standard.

Phospholipid fatty acids are named by standard nomenclature: the total number of carbon atoms, followed by a colon and the number of double bonds. The position of the first double bond is indicated by " $\omega$ " followed by the number of carbon atoms counted from the aliphatic end. The suffixes $\mathrm{c}$ for cis and $\mathrm{t}$ for trans refer to geometric isomers. The prefixes "a" and "i" refer to anteiso- and iso-branched fatty acids. The prefix "10Me" indicates a methyl group on the tenth carbon atom from the carboxyl end of the molecule. The prefix "cy" denotes cyclopropane fatty acids. To estimate proportions of the main taxa of the microbiota in samples by PLFAs, the branched, saturated 
PLFAs were chosen to represent Gram-positive (GP) bacteria (O'Leary and Wilkinson, 1988), and the monoenoic and cyclopropane unsaturated fatty acids represent Gramnegative (GN) bacteria (Wilkinson, 1988). The unsaturated PLFA 18:2 $\omega 6,9$ was used as an indicator of fungal biomass (Johansen and Olsson, 2005).

\subsection{T-RFLP analysis of 16S rDNA genes}

Phyllosphere microorganism metagenomic DNA from the controls and cypermethrin treatments was extracted using Yang's method (Yang et al., 2001). The DNA was purified using a DNA Gel Recovery Kit (Omega Bio-Tek, Inc., USA). The primers used for the PCR amplification of 16S rDNA sequences were 27F (5'-AGAGTTTGATCCTGGCTCAG-3') and 1492R (5'-GGTTACCTTGTTACGACTT-3') (Blackwood et al., 2003). 27F was labeled at the 5' end with the 6'carboxyfluorescein (6-FAM), which was synthesized by Shanghai GeneCore Biotechnologies Co., Ltd., China. Amplification reactions were performed in a total volume of $50 \mu \mathrm{l}$ containing $3 \mu \mathrm{l}$ dissolved DNA (50 ng), PCR buffer $(10 \mathrm{mmol} / \mathrm{L}$ Tris-HCl, pH 9, $50 \mathrm{mmol} / \mathrm{L} \mathrm{KCl}, 0.1 \%$ Triton X-100, and $2 \mathrm{mmol} / \mathrm{L} \mathrm{MgCl}_{2}$ ), $200 \mathrm{mmol} / \mathrm{L}$ of each deoxynucleoside triphosphate, $100 \mathrm{nmol} / \mathrm{L}$ of each primer, and $2.5 \mathrm{U}$ of TaKaRa Taq DNA polymerase. Thermal cycling conditions were as follows: 5 min denaturation at $94^{\circ} \mathrm{C}$ followed by 30 cycles of denaturation at $94^{\circ} \mathrm{C}$ for $1 \mathrm{~min}$, annealing at $55^{\circ} \mathrm{C}$ for $30 \mathrm{~s}$, and extension at $72^{\circ} \mathrm{C}$ for $45 \mathrm{~s}$, and a final extension for $3 \mathrm{~min}$ at $72^{\circ} \mathrm{C}$. Three replicate reactions were run for each sample and the products were pooled.

The PCR products were examined in a $1 \%$ agarose gel and subsequently purified with an Omega PCR purification kit (Omega Bio-Tek, Inc., USA). Purified PCR products (approximately $200 \mathrm{ng}$ ) were digested using $10 \mathrm{U}$ of tetrameric restriction endoncleases RsaI (NEB) for 16$18 \mathrm{~h}$ at $37^{\circ} \mathrm{C}$ in a $20-\mu \mathrm{l}$ reaction volume. The samples were separated with a GeneScan 1000 Rox (Applied Biosystems) as an internal size standard on an ABI 310 DNA sequencer (Applied Biosystems) using POP6 poly- mer. Terminal fragments were evaluated using a GeneScan Analytical Software version 3.1.2. T-RFLP profiles in the range of 40-500 bp were used for the analysis.

\subsection{Data analysis}

Data were presented as means together with standard error (SE). Fatty acids with carbon lengths of 14-22 were used to analyze microbial community structure. Principalcomponent analyses were performed to compare the PLFA profiles in the samples. Fatty acids were transformed to their mole fractions. SPSS 11.5 software (version 13.0, SPSS Inc., USA) was used to perform PCA. For T-RFLP data, a binary matrix was constructed considering the presence or absence of a peak in the profile. The binary matrix profiles were then compared to compute the community similarity values using the Dice index as implemented in the software NTSYSpc 2.0 (Rohlf, 1990). The matrix of Dice similarity values was then used for construction of a UPGMA (unweighted pairs group method with arithmetic average) dendrograms algorithm with bootstrap values over 1,000 replicas using the modules present in NTSYSpc 2.0 .

\section{Results}

\subsection{PLFA analysis}

The total amount of PLFAs can be used to indicate the total microbial biomass. Analysis of PLFA profiles of control and cypermethrin treatments on day $0,1,3$, 6,10 , and 15 was carried out in triplicate. The results in Fig.1a showed that there was a significant impact of cypermethrin treatments on the total PLFA concentration of cucumber phyllosphere microorganism. The total PLFA was higher in cypermethrin treated phyllosphere than this analyzed in control through the experiment. For the cypermethin treated phyllosphere samples, the highest total PLFA concentration occurred on day 6 and then the PLFA concentrations declined. Total bacterial PLFA concentrations followed similar patterns as total PLFA
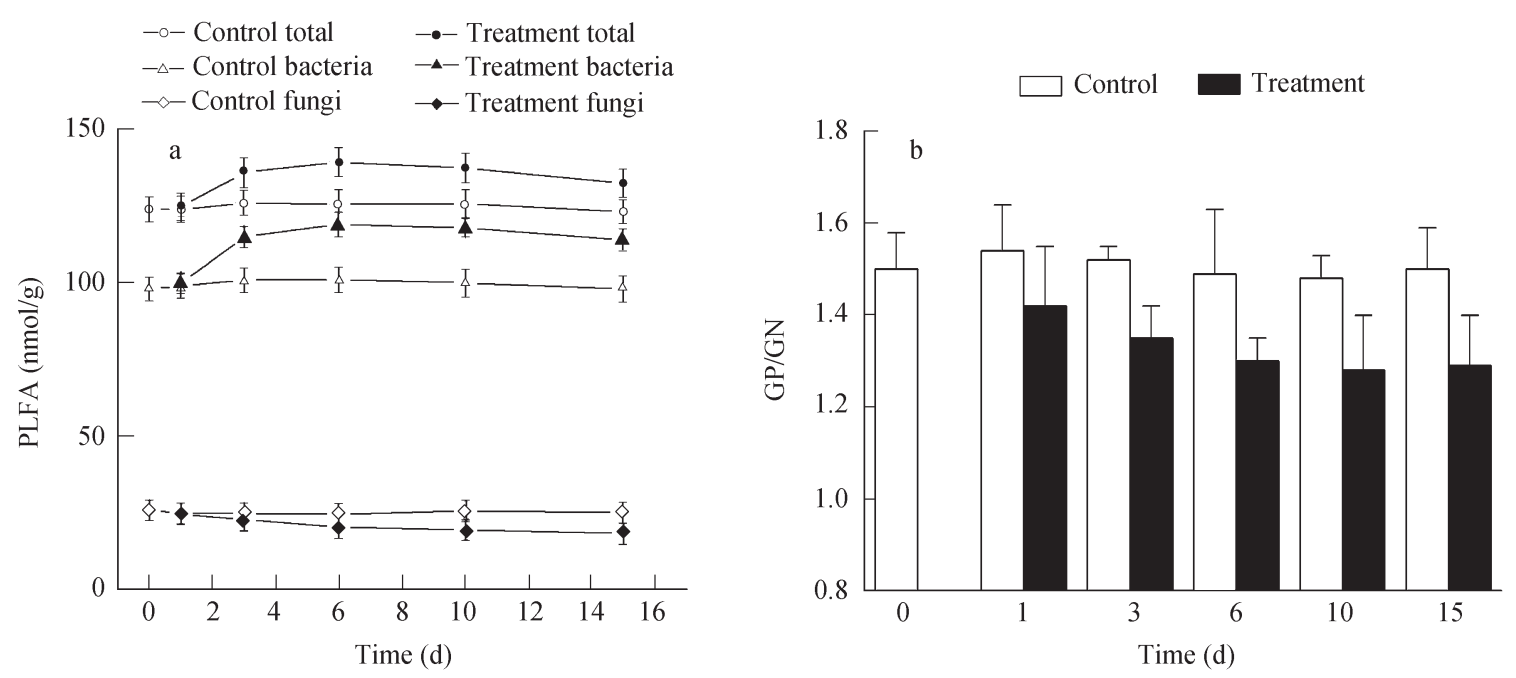

Fig. 1 Fifteen-day response of (a) total PLFA, bacteria PLFA, fungal PLFA, and (b) GP/GN PLFA to cypermethrin in cucumber phyllosphere. Error bar represent the standard error of the mean $(n=3)$. 
during the $15 \mathrm{~d}$ experiment (Fig.1a). However, the fungal PLFA concentration in cypermethrin treated phyllosphere was lower than that in control phyllosphere (Fig.1a). The lowest fungal PLFA reached a concentration of 18.4 $\mathrm{nmol} / \mathrm{g}$ sample on day 15 . The ratio of Gram-positive (GP) bacteria to Gram-negative (GN) bacteria was also affected by cypermethrin treatment. GP/GN was found to be lower in the cypermethrin treated phyllosphere than these in control (Fig.1b).

Principal-component analyses were performed to find out variations in major microbial groups within the phyllosphere on the cypermethrin-treated and control plants at different times. Phospholipid fatty acid patterns from cypermethrin treatment plots were clearly separated from the control plots along PC1, and scores from the treatment coordinated to the right in the score plot along PC1, whereas the control ones were located to the left (Fig.2a). Very similar results along PC1 were found from the cypermethrin-treated samples during the growth period, except the sample on day 1, but showed some variation along PC2. Hence, succession time (represented by PC2) was the factor responsible for the variation in the cypermethrin-treated phyllosphere microbial community. The loadings in Fig.2b showed that PC1 had a clear positive relationship with the following six PLFA species: the saturated PLFA i16:0, i17:0; the unsaturated PLFA

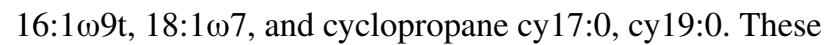
PLFA species were found in the right part of the loading plot, representing the charges of the cypermethrin-treated phyllosphere microbial community. The remaining 12 PLFA species, which were more associated with the control phyllosphere microbial community, clustered together in the left part of the loading plot. Along PC2 axis, the unsaturated PLFA 16:1 $\omega 9 \mathrm{t}, 18: 1 \omega 7$, and cyclopropane cy 17:0, cy19:0 were positively correlated with PC2, where the saturated PLFA i16:0, i17:0 were negatively correlated with PC2. The result indicated that the number of unsaturated and cyclopropane PLFAs increased after using the cypermethrin treatment, whereas the number of the saturated PLFA i16:0, i17:0 decreased.

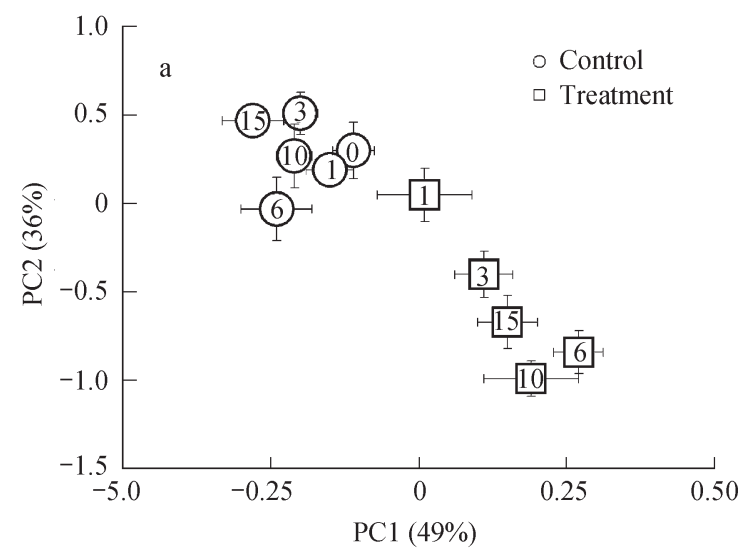

\subsection{T-RFLP analysis}

Genetic fingerprinting with T-RFLP of 16S rDNA was used to determine the effects of cypermethrin treatment on phyllosphere microbial communities. The frequency of the most abundant terminal restriction fragments (TRFs) obtained from the control and cypermethrin-treated samples is shown in Fig.3. The T-RFLP analyses revealed some significant differences in the number and types of TRFs observed for both control and treatments. Although we noted that several of the T-RFs $(47,66,204,236$, 420 , and $482 \mathrm{bp)}$ appeared in all samples, there were also obvious changes in presence and absence of T-RFs due to the treatments and times effected (see the number of occurrences in Fig.3). Four T-RFs of 51, 303, 331, and 338 bp were present in control but absent in all cypermethrintreated samples. Similarly, the four other fragments (44, 96, 223, and $306 \mathrm{bp}$ ), which were only seen in control samples, were missing in exposure to cypermethrin after 1 day. However, some fragments $(58,62,89,99,119,195$, 239311,340 , and $473 \mathrm{bp}$ ) were not found in all control but appeared in three or more of the treatments.

Dendrogram analysis (Fig.4) of the T-RFLP profiles confirms that exposure to cypermethrin insecticide had a significant impact on the phyllosphere microbial community structure. Two main clusters of profiles were differentiated at low similar values (less than 46\%). The first cluster included the profiles of all of the samples from control and the treatment in the 1st day. The second cluster grouped the profiles of nearly all sample treated with cypermethrin expect on day 1 . The results indicated that there were changes in phyllosphere microbial communities on day 1 after the treatments with cypermethrin insecticide. Another observation is that the effect of time on bacterial community structure was also evidenced by the grouping of profiles according to sampling data within clusters I and II in Fig.4. In the cluster I, three smaller clusters separated the samples according to the sampling data and the treatments $(1 \mathrm{~d})$.

In the case of cluster II, the phyllosphere bacteria communities after cypermethrin application also showed some changes. The samples on day 3 and 6 produced

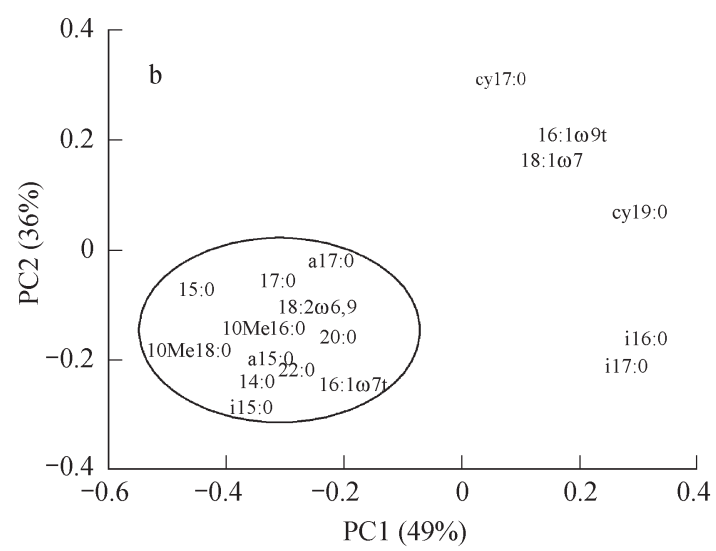

Fig. 2 Scatter plot of the score (a) and loading (b) values of the first two principal components in a principal component analysis of relative molar abundance of fatty acids originating from the phospholipids in the total microbial community in cucumber phyllosphere. The day after treatment is shown in the individual marker. 


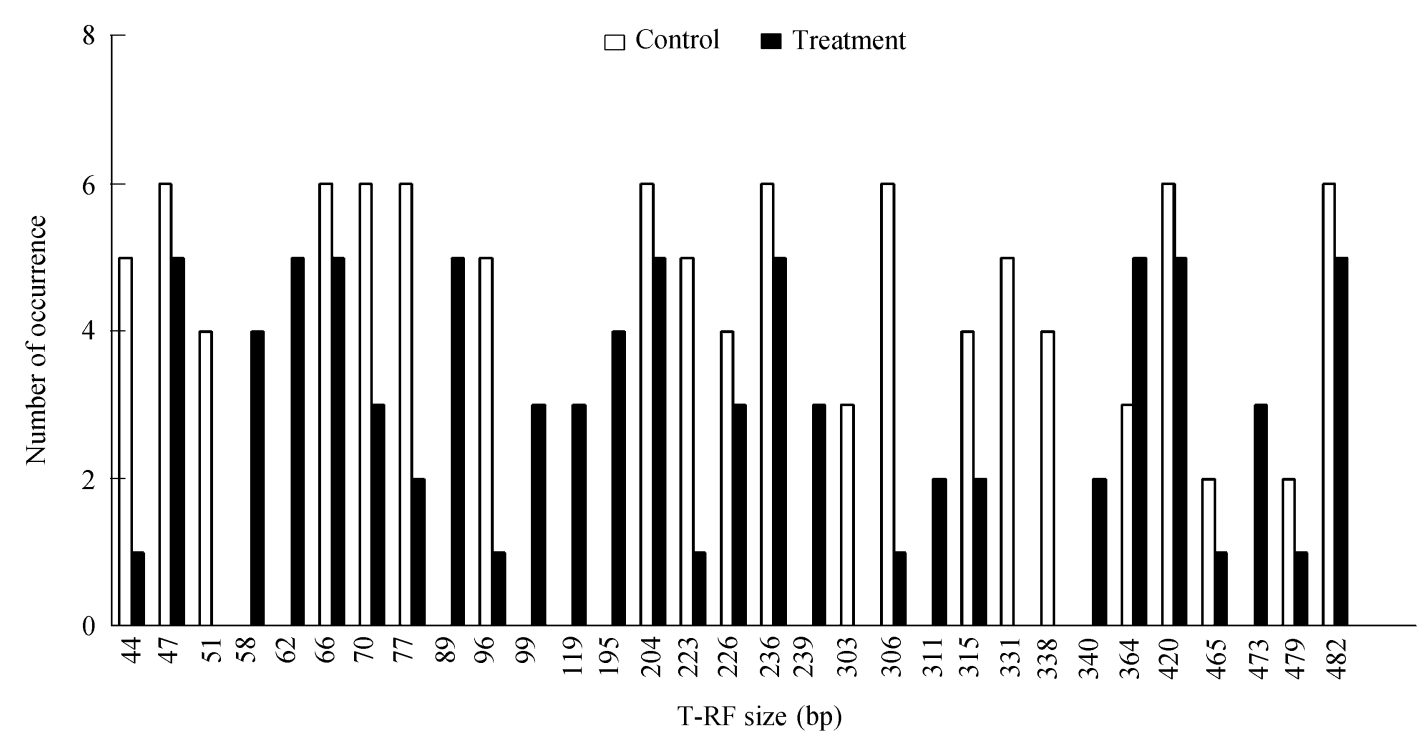

Fig. 3 Frequency plot of the individual T-RFs generated from different samples including six controls and five cypermethrin treatments. Note that the horizontal scale is not linear due to the omission of T-RFs sizes with zero or one occurrence.

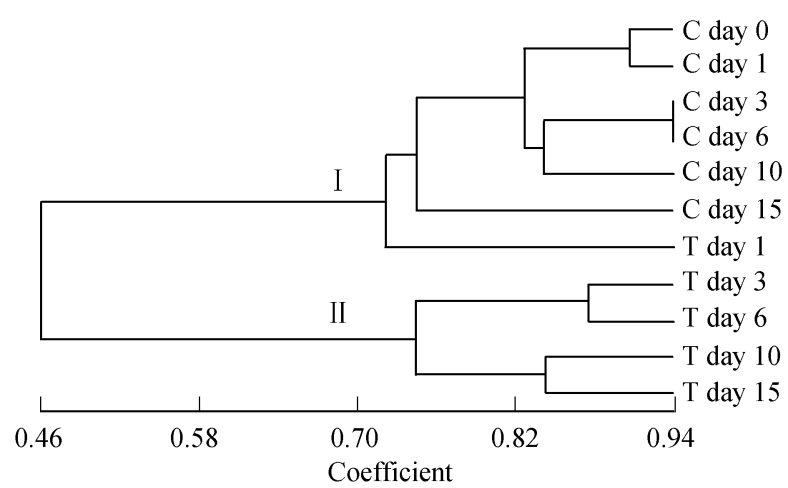

Fig. 4 UPGMA dendrogram computed from Dice similarity matrix among T-RFLP profiles of amplified 16S rDNA gene. Scale bar indicates Dice similarity values. C: Control; T: Treatment.

the similar profiles (average similarity about $90 \%$ ). The samples on day 10 and 15 clustered together (average similarity about $85 \%$ ), which separated from the other.

\section{Discussion}

A few recent studies have used the culture-independent methods to study contaminant effects on microbial communities of plants phyllosphere. This study was the first attempt to assess the impact of pyrethroid insecticide cyperemethrin on microbial communities in plant phyllosphere using biochemical and genetic approaches. Phyllosphere microbial communities provide important function in aerial ecosystems of the plants. Meanwhile, phyllosphere microbial communities are also affected by different environmental conditions. For example, rainfall and wetting could change the phyllosphere community of the resurrection fern (Jackson et al., 2006). Kadivar and Stapleton (2003) found that ultraviolet radiation can significantly alter maize phyllosphere bacterial community structure. Green et al. (2006) showed that differential effects of urea application on cherry leaf litter were found within groups of both bacteria and fungi. Our study clearly showed that the application of pyrethroid insecticide positively affected some phyllosphere microbial activities and changed microbial community structures.

The composite PLFA profiles provided an indication of phyllosphere microbial biomass and, in turn, community structure. Microbial biomass can be measured by summation of the mass of all detectable PLFAs (Ben-David et al., 2004). Total PLFAs, especially for bacterial PLFAs, were significantly increased compared with the control (Fig.1a). The reason may be that cypermethrin insecticide likely stimulated some bacterial groups, which could use the insecticide as a carbon source for growth. Several studies have also shown the similar results. For example, Diğrak and Kazanici (2001) reported that the total number of viable bacteria was found to be higher than that of the control groups during incubation in the isofenphostreated soil sample. Chinalia and Killham (2006) found that bacterial biomass of sediment microcosms responded with an increase in 2,4-D inputs. The PLFA data also suggested that the cypermethrin insecticide treatment resulted in an increase in GN bacteria (Fig.1a). An increase in GN bacteria PLFA indicated that these GN bacteria might have more tolerance for the insecticide in the cucumber phyllosphere. Furthermore, a decrease in fungi PLFA was observed at the insecticide treatment sampling. This is most likely due to the fact that fungi tend to be more sensitive to cypermethrin insecticide than bacteria.

The distribution of the treatments and controls in the score plots in the PCA of the PLFAs gave information about how the cypermethrin insecticide affected the phyllosphere microbial community structure. Our PLFA-data from treatment samples revealed a close association with

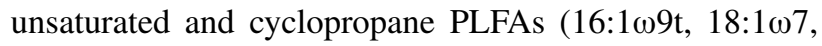
cy 17:0, cy 19:0) indicative of GN bacteria (Fig.2b). Fang et

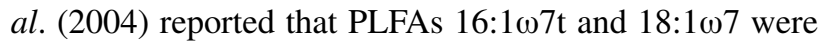
biomarker of pseudomonad group. These results suggested that those bacteria might play significant roles in the 
degradation of cypermethrin insecticide in the cucumber phylosphere. Recently, Pseudomonas sp. had been isolated from other environment as a type of cypermethrin degrader (Jilani and Khan, 2006). However, Pseudomonas sp. is not reported in the literature as an organism responsible for cypermethrin insecticide biodegradation in the plants phyllosphere.

The T-RFLP analysis has mainly been applied as a tool for the comparison of microbial communities. Cypermethrin-induced change in microbial community structure was also detected by the presence and absence of T-RFs on our T-RFLP analysis. A total of 31 different T-RFs were detected in the present study (Fig.3). Although the average number of T-RFs per sample were similar (data no shown), the types of T-RFs were found to be significantly different between the controls and treatments. Exposure of cypermethrin insecticide caused the appearance of some "new" fragments that were missing in controls or the disappearance of one or more T-RFs. These T-RFs might perform critical ecological function. The "new" T-RFs (58, 62, 89, 99, 119, 195, 239 311, 340, and $473 \mathrm{bp})$, which could represent some bacteria, might play a significant role in the degradation of cypermethrin insecticide in the cucumber phylosphere. The "disappeared" T-RFs (51, 303, 331, and $338 \mathrm{bp})$ indicating the other different bacteria might potentially serve as microbial indicator of the insecticide exposure. According to Dice's coefficient of similarity in the microbial community structure of different microcosms by cluster analysis, two major clusters can be formed (Fig.4). The T-RFLP data, here, reinforced the PLFA results that application cypermethrin insecticide can change cucumber phyllosphere microbial community structure. Interestingly, the T-RFLP analyses identified the community compositions for treated sample on day 3 and 6 to be the most similar (Fig.4), whereas the PLFA data identified the phyllosphere community for sample on day 3 and 15 as being most similar (Fig.2a). The major bias attributed to the difference between T-RFLP and PLFA results was due to the differences in approaches between biochemical and genetic-based assays.

This study is the first using PLFA and T-RFLP to investigate the effects of insecticide application on plant phyllosphere microbial community. An advantage of PLFA analysis is that it can provide quantitative insight in effect on major microbial groups. The T-RFLP analysis, on the other hand, can generate a high throughput and reproducibility in monitoring microbial communities. Future studies will investigate the influence of different pesticides upon the composition and structure of phyllosphere microbial communities for different plants. In turn, a better understanding will be made regarding how the microbial communities are responsible for the degradation of the pesticides.

\section{Acknowledgments}

This work was supported by the National Natural Science Foundation of China (No. 30600082) and the Australian Research Council (ARC) International Linkage Fellowship (No. LX0560210).

\section{References}

Amann R I, Ludwig W, Schleifer K H, 1995. Phylogenetic identification and in situ detection of individual microbial cells without cultivation. Microbiol Rev, 59: 143-169.

Ben-David E, Holden P J, Stone D J M, Harch B D, Foster L J, 2004. The use of phospholipid fatty acid analysis to measure impact of acid rock drainage on microbial communities in sediments. Microbl Ecol, 48: 300-315.

Blackwood C B, Marsh T, Kim S H, Paul E A, 2003. Terminal restriction fragment length polymorphism data analysis for quantitative comparision of microbial communoities. Appl Environ Microbiol, 69: 926-932.

Chinalia F A, Killham K S, 2006. 2,4-Dichlorophenoxyacetic acid (2,4-D) biodegradation in river sediments of NortheastScotland and its effect on the microbial communities (PLFA and DGGE). Chemosphere, 64: 1675-1683.

Diğrak M, Kazanici F, 2001. Effect of some organophosphorus insecticides on soil microorganisms. Turk J Biol, 25: 5158.

Fang J, Lovanh N, Alvarez P J, 2004. The use of isotopic and lipid analysis techniques linking toluene degradation to specific microorganisms: applications and limitations. Water Res, 38: 2529-2536.

Green H, Bengtsson M, Duval X, Pedersen H L, Hockenhull J, Larsen J, 2006. Influence of urea on the cherry leaf spot pathogen, Blumeriella jaapii, and on microorganisms in decomposing cherry leaves. Soil Biol Biochem, 38: 27312742.

Hirano S S, Upper C D, 2000. Bacteria in the leaf ecosystem with emphasis on Pseudomonas syringae - a pathogen, ice nucleus, and epiphyte. Microbiol Mol Biol Rev, 64: 624653.

Hiraishi A, Iwasaki M, Shinjo H, 2000. Terminal restriction pattern analysis of 16S rRNA gene for the characterization of bacterial communities of activated sludge. $J$ Biosci Bioengin, 90: 147-156.

Jackson E F, Echlin H L, Jackson C R, 2006. Changes in the phyllosphere community of the resurrection fern, Polypodium polypodioides, associated with rainfall and wetting. FEMS Microbiol Ecol, 58: 236-246.

Jilani S, Khan M A, 2006. Biodegradation of cypermethrin by Pseudomonas in a batch activated sludge process. Int $J$ Environ Sci Technol, 3: 371-380.

Johansen A, Olsson S, 2005. Using phospholipid fatty acid technique to study short-term effects of the biological control agent Pseudomonas fluorescens DR54 on the microbial microbiota in barley rhizosphere. Microb Ecol, 49: 272281.

Kadivar H, Stapleton A E, 2003. Ultraviolet radiation alters maize phyllosphere bacterial diversity. Microb Ecol, 45: 353-361.

Ko W, Lockwood J L, 1968. Conversion of DDT to DIDD in soil and the effect of these compounds on soil microorganisms. Can J Microbiol, 14: 1069-1073.

Krechel A, Faupel A, Hallmann J, Ulrich A, Berg G, 2002. Potato-associated bacteria and their antagonistic potential towards plant-pathogenic fungi and the plant-parasitic nematode Meloidogyne incognita (Kofoid \& White) Chitwood. Can J Microbiol, 48: 772-786.

Krimm U, Abanda-Nkpwatt D, Schwab W, Schreiber L, 2005. Epiphytic microorganisms on strawberry plants (Fragaria ananassa cv. Elsanta): identification of bacterial isolates and analysis of their interaction with leaf surfaces. FEMS Microbiol Ecol, 53: 483-492. 
Kvasnikov E I, Kliushnikova T M, Nagornaia S S, 1974. The nitrogen fixing ability of yeasts isolated from the rhizosphere and phyllosphere of plants. Mikrobiol Zh, 36: 790-791.

Lal R, Saxena D M, 1982. Accumulation, metabolism, and effects of organochlorine insecticides on microorganisms. Microbiolo Res, 46: 95-127.

Lindow S E, Leveau J H, 2002. Phyllosphere microbiology. Curr Opin Biotechnol, 13: 238-243.

Lindow S E, Brandl M T, 2003. Microbiology of the phyllosphere. Appl Environ Microbiol, 69: 1875-1883.

Murty M G, 1984. Phyllosphere of cotton as a habitat for diazotrophic microorganisms. Appl Environ Microbiol, 48: 713-718.

O'Leary W, Wilkinson S G, 1988. Gram-positive bacteria. In: Microbial Lipids (Ratledge C., Wilkinson S. G., eds.). London: Academic Press. Vol 1. 117-201.

Pandey P, Chauhan U K, 2007. Effects of pesticides (endo sulfan and quinal phos) on microbial community under controlled conditions. FASEB J, 21: 791.2.

Pang C M, Liu W T, 2007. Community structure analysis of reverse osmosis membrane biofilms and the significance of rhizobiales bacteria in biofouling. Environ Sci Technol, 41: 4728-4734.

Pennanen T, Liski J, Baath E, Kitunen V V, Uotila J, Westman C J, Fritze H, 1999. Structure of the microbial communities in coniferous forest soils in relation to site fertility and stand development stage. Microb Ecol, 38: 168-179.

Ratcliff A, Busse M D, Shestak C J, 2006. Changes in microbial community structure following herbicide (glyphosate) additions to forest soils. Appl Soil Ecol, 34: 114-124.

Rohlf F J, 1990. NTSYS-pc. Numerical taxonomy and multivariate analysis system. Version 2.02. Exeter Software, New York.
Salonlus P, 1972. Effect of DDT and fenitrothion on forest soil microflora. J Econ Entomol, 65: 1089-1096.

Sandhu A, Halverson L J, Beattie G A, 2007. Bacterial degradation of airborne phenol in the phyllosphere. Environ Microbiol, 9: 383-392.

Schreiber L, Krimm U, Knoll D, Sayed M, Auling G, Kroppenstedt R M, 2005. Plant-microbe interactions: identification of epiphytic bacteria and their ability to alter leaf surface permeability. New Phytol, 166: 589-594.

Lenteren J C, 2000. A greenhouse without pesticides: fact or fantasy? Crop Protection, 19: 375-384.

Vig K, Singh D K, Agarwal H C, Dhawan A K, Dureja P, 2008. Soil microorganisms in cotton fields sequentially treated with insecticides. Ecotoxicol Environ Saf, 69: 263-276.

Walter M, Frampton C M, Boyd-Wilson K S, Harris-Virgin P, Waipara N W, 2007. Agrichemical impact on growth and survival of non-target apple phyllosphere microorganisms. Can J Microbiol, 53: 45-55.

Wilkinson S, 1988. Gram-negative bacteria. In: Microbial Lipids (Ratledge C., Wilkinson S. G., eds.). London: Academic Press. Vol 1. 299-448.

Xue K, Luo H F, Qi H Y, Zhang H X, 2005. Changes in soil microbial community structure associated with two types of genetically engineered plants analyzing by PLFA. J Environ Sci, 17(1): 103-134.

Yang C H, Crowley D E, Borneman J, Keen N T, 2001. Microbial phyllosphere populations are more complex than previously realized. Proc Natl Acad Sci USA, 98: 3889-3894.

Zhang Z Y, Liu X J, Yu X Y, Zhang C Z, Hong X Y, 2007. Pesticide residues in the spring cabbage (Brassica oleracea L. var. capitata) grown in open field. Food Control, 18: 723730 . 